\title{
The specificity of SNARE pairing in biological membranes is mediated by both proof-reading and spatial segregation
}

\author{
loanna Bethani ${ }^{1,2}$, Thorsten Lang ${ }^{1}$, \\ Ulf Geumann ${ }^{1}$, Jochen J Sieber ${ }^{1}$, \\ Reinhard Jahn ${ }^{1, *}$ and Silvio O Rizzoli ${ }^{1}$
}

\author{
${ }^{1}$ Department of Neurobiology, Max-Planck-Institute for Biophysical \\ Chemistry, Göttingen, Germany and ${ }^{2}$ International Max Planck Research \\ School Neurosciences, Max-Planck-Institute for Biophysical Chemistry, \\ Göttingen, Germany
}

Soluble $\mathrm{N}$-ethylmaleimide-sensitive factor attachment receptor (SNARE) proteins mediate organelle fusion in the secretory pathway. Different fusion steps are catalyzed by specific sets of SNARE proteins. Here we have used the SNAREs mediating the fusion of early endosomes and exocytosis, respectively, to investigate how pairing specificity is achieved. Although both sets of SNAREs promiscuously assemble in vitro, there is no functional crosstalk. We now show that they not only colocalize to overlapping microdomains in the membrane of early endosomes of neuroendocrine cells, but also form cis-complexes promiscuously, with the proportion of the different complexes being primarily dependent on mass action. Addition of soluble SNARE molecules onto native membranes revealed preference for cognate SNAREs. Furthermore, we found that SNAREs are laterally segregated at endosome contact sites, with the exocytotic synaptobrevin being depleted. We conclude that specificity in endosome fusion is mediated by the following two synergistically operating mechanisms: (i) preference for the cognate SNARE in 'trans' interactions and (ii) lateral segregation of SNAREs, leading to relative enrichment of the cognate ones at the prospective fusion sites.

The EMBO Journal (2007) 26, 3981-3992. doi:10.1038/

sj.emboj.7601820; Published online 23 August 2007

Subject Categories: membranes \& transport

Keywords: cis-complex; endosome; fusion; SNARE trans-complex

\section{Introduction}

Soluble $N$-ethylmaleimide-sensitive factor attachment receptor (SNARE) proteins are widely regarded as major players in the fusion of intracellular membranes. They constitute a superfamily of membrane-associated proteins characterized by the presence of at least one SNARE motif, which is typically found in the immediate vicinity of the transmembrane domain

\footnotetext{
${ }^{*}$ Corresponding author. Department of Neurobiology, Max-PlanckInstitute for Biophysical Chemistry, Am Fassberg 11, Göttingen 37077, Germany. Tel.: + 49551201 1635; Fax: +49 551201 1639;

E-mail: rjahn@gwdg.de
}

Received: 6 February 2007; accepted: 19 July 2007; published online: 23 August 2007 of the protein. SNARE motifs are classified into four subfamilies, termed Qa-, Qb-, Qc- and R-SNAREs. To catalyze fusion, the SNAREs from two apposing membranes interact in a 'trans'-configuration. Their SNARE motifs assemble into bundles of four $\alpha$-helices, and each bundle invariantly contains one SNARE motif of each subfamily. Bundle formation is initiated at the N-terminal end of the SNARE motif, and continues toward the C-terminal end (toward the membrane), thus bringing the two membranes together. After fusion, all members of the complex are found in the same membrane ('cis'-configuration). Disassembly of the complex requires the activity of the AAA-ATPase NSF that separates the monomers and allows them to engage in subsequent fusion steps (see reviews in Jahn et al, 2003; Hong, 2005; Jahn and Scheller, 2006).

Different SNAREs seem to participate in fusion events involving different processes and organelles. It is generally accepted that each fusion step of the secretory pathway requires a specific set of SNAREs, although some SNAREs are known to operate in different fusion steps and others are able to substitute for each other. However, it is unclear by which mechanisms it is ensured that only 'cognate' SNAREs interact with each other for fusion. SNAREs associate in vitro with little discrimination between cognate and non-cognate sets, as long as a member of each subclass is present (see review by Jahn and Scheller, 2006). Similar promiscuity was observed in in vitro fusion assays when mammalian SNAREs were reconstituted in artificial vesicles (Brandhorst et al, 2006).

The question how cognate SNAREs are selected for fusion is particularly relevant for recycling compartments such as the early endosomes, which constitute a major hub in the endocytotic limb of the secretory pathway. Early endosomes communicate with the trans-Golgi network, with recycling endosomes and late endosomes (Maxfield and McGraw, 2004). Consequently, they contain not only SNAREs mediating endosome fusion, but also additional sets of SNAREs that are 'passengers' en route to their resident compartment, including those mediating exocytosis and fusion of late endosomes (Brandhorst et al, 2006). Despite being present in the same membrane, the different sets of SNAREs are functionally well differentiated in the respective fusion steps. For instance, fusion of early endosomes can be competed for by the soluble parts of the cognate, but not of the neuronal SNAREs (Brandhorst et al, 2006). Conversely, cleavage by botulinum or tetanus neurotoxin of any of the neuronal SNAREs blocks synaptic vesicle fusion (see review by Humeau et al, 2000), although the endosomal SNAREs are present on these vesicles (Antonin et al, 2000; Rizzoli et al, 2006).

In the present study, we have used a combination of approaches in order to shed light on the mechanisms that govern cognate and non-cognate SNARE interactions in native and fusion-competent membranes. Using early endosomal and neuronal SNAREs as example, we find that within the endosomal membrane, these SNAREs not only are 
concentrated in overlapping microdomains, but are able to promiscuously form cognate and non-cognate cis-complexes. The proportions of the cognate and non-cognate complexes are dependent on the relative amounts of the individual SNAREs and, as revealed by simulations, are primarily being determined by mass action. When probing for transSNARE interactions by addition of soluble SNARE molecules onto native membranes, a certain level of specificity was uncovered, as shown by a preferential binding of cognate SNAREs to their SNARE partners in native membranes. Finally, we observed that at the contact site of fusing endosomes there is lateral segregation of SNAREs, with the exocytotic SNARE synaptobrevin being depleted. We conclude that there is little specificity in complex formation for SNAREs residing in the same membrane, and thus many published SNARE complexes that were previously identified by coprecipitation may not represent functional sets of SNAREs. Trans-specificity is mediated (i) by a preference (but not absolute specificity) for the cognate SNARE in the trans-configuration, probably involving assistance by proofreading proteins such as SM-proteins, and (ii) by lateral segregation of SNAREs at the contact site of fusing organelles.

\section{Results}

\section{Cognate and non-cognate SNAREs accumulate in common microdomains on the endosomal membrane}

Early endosomes of neuroendocrine PC12 (pheochromocytoma) cells contain both the SNAREs mediating homotypic fusion (including VAMP4 (R), syntaxin 13 (Qa), syntaxin 6 $(\mathrm{Qb})$ and vtila (Qc)) and the SNAREs mediating regulated exocytosis of secretory vesicles (including synaptobrevin/ VAMP2 (R), syntaxin 1 (Qa) and SNAP-25 (Qbc)). Selective cleavage of the three exocytotic SNAREs blocks exocytosis (Humeau et al, 2000), showing that the early endosomal SNAREs cannot substitute for their exocytotic counterparts in this reaction. Conversely, cleavage of SNAP-25 with BoNT/E, or of syntaxin 1 with BoNT/C1 does not inhibit fusion of early endosomes (Brandhorst et al, 2006; Rizzoli et al, 2006). Cleaving the remaining exocytotic SNARE (synaptobrevin) with tetanus neurotoxin also had no effect on fusion (Supplementary Figure 1). Thus, there is no functional crosstalk between exocytotic and endosomal SNAREs.

Although both sets of SNAREs share the same membrane, it is conceivable that their interaction is prevented by lateral segregation in separate microdomains. Such segregation has recently been observed for plasma membrane-resident syntaxins that participate in separate SNARE complexes (Low et al, 2006; Sieber et al, 2006). Since early endosomes are too small to allow discrimination of microdomains by conventional light microscopy, we transfected PC12 cells with a constitutively active mutant of the endosomal GTPase Rab5 (Rab5-Q79L) that was tagged with GFP. Very large endosomes are generated due to increased homotypic fusion activity (Stenmark et al, 1994), which are imaged easily (Figure 1A). The cells were then immunostained for pairs of both cognate and non-cognate SNAREs (Figure 1B-D). An uneven, punctate staining pattern was obtained that was similar between the two SNAREs and contrasted with the more even distribution of GFP-Rab5-Q79L (Figure 1E). To score for colocalization between different SNAREs, we performed confocal sections around the equator of the
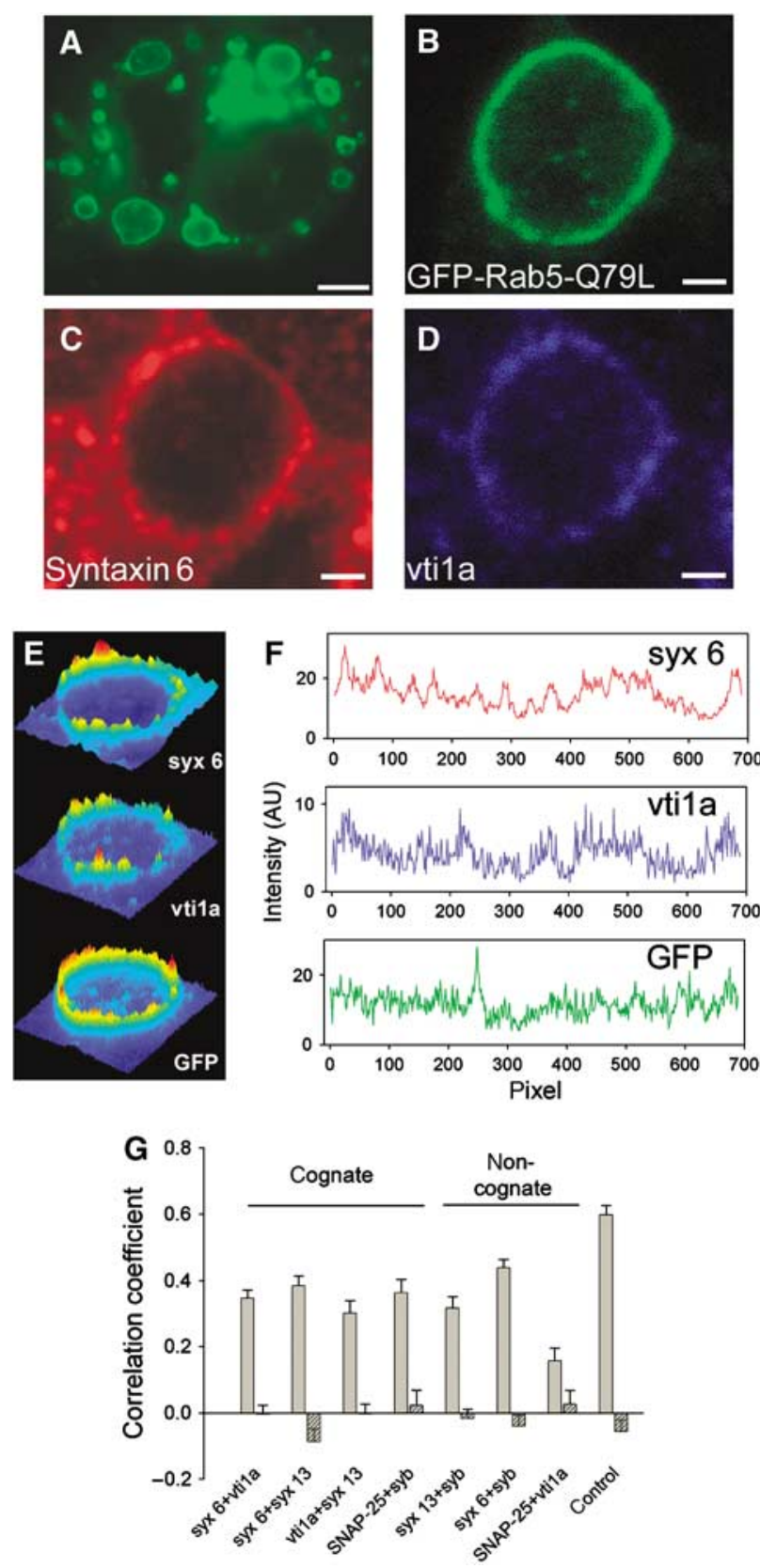

Figure 1 The cognate and non-cognate SNAREs largely colocalize in microdomains on the endosomal membrane. (A) PC12 cells were transfected with a plasmid expressing GFP-Rab5-Q79L. Forty-eight hours post-transfection, cells were fixed and imaged by use of a Zeiss Axiovert 200M fluorescence microscope. The GFP-bound Rab5 variant was observed on vesicular structures of $1-5 \mu \mathrm{m}$, which correspond to enlarged early endosomes. Scale bar, $5 \mu \mathrm{m}$. (B-D) PC12 cells expressing the GFP-Rab5-Q79L (green) were stained for syntaxin 6 (red channel) and vtila (blue channel), and imaged by confocal fluorescence microscopy. Images show a typical endosome. Note the SNARE domains on the endosomal membrane. Scale bar, $1 \mu \mathrm{m}$. (E) The intensity images in panels B-D are plotted as surfaces, in pseudocolor. Note that the SNAREs are found in domains, which largely correlate. (F) Intensity profiles of syntaxin 6 (red), vti1a (blue) and GFP-Rab5-Q79L (green) signals along the endosomal membrane of the endosome shown in panels B-E. (G) Correlation between the staining of different pairs of SNAREs on the endosomal membrane. Control, costaining of single SNAREs with Cy3- and Cy5-conjugated secondary antibodies. Values are mean$\mathrm{s} \pm$ s.e. of three independent experiments with 10-15 analyzed endosomes. The striped bars correspond to the negative control (see Materials and methods). 
endosomes, followed by cross-correlation analysis along the endosomal membrane (Figure $1 \mathrm{~F}$; see Materials and methods). All SNARE pairs investigated showed a high degree of cross-correlation (although somewhat less for the SNAP-25/vtila pair), documenting that within the resolution limits of the confocal microscope, there is no appreciable difference in localization between cognate and non-cognate SNARE pairs (Figure 1G).

\section{Cognate and non-cognate SNAREs form complexes in the membrane of early endosomes}

Considering that the neuronal SNAREs play no role in endosome fusion, although they are concentrated together with their endosomal counterparts in overlapping microdomains, the question arises whether these SNAREs can form promiscuous complexes (as they do in vitro) or whether native membranes possess mechanisms preventing such non-cognate interactions. To answer this question, we purified early endosomes by discontinuous sucrose gradient centrifugation, resulting in high enrichment of endosomal markers such as Rab5 (Figure 2A), followed by solubilization in Triton X-100. Synaptobrevin was then immunoprecipitated, and the precipitate was analyzed for the presence of both neuronal and early endosomal SNAREs. The results are shown in Figure 2B-C. Both endosomal (syntaxin 6, syntaxin 13, vtila) and neuronal (SNAP-25 and syntaxin 1) Q-SNAREs were identified
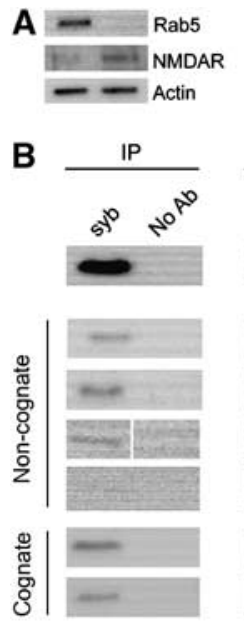

Supernatant
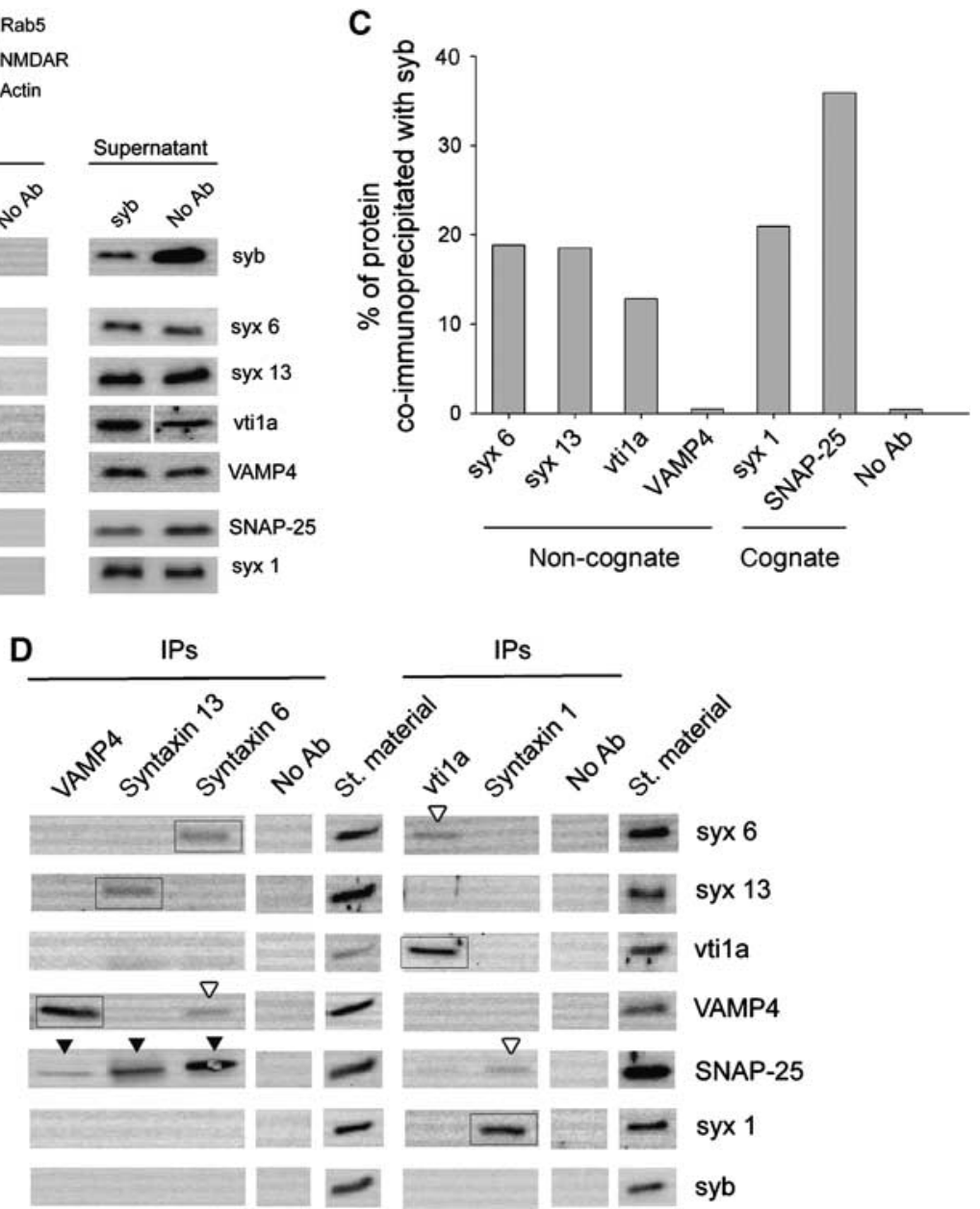

Figure 2 Interactions between non-cognate SNAREs in early endosomes. (A) Enrichment of an early endosomal-specific marker (Rab5) in the fraction isolated from the gradient. Equal amounts of the endosomal fraction (lane1) and post nuclear supernatant (PNS) (lane 2) were analyzed by SDS-PAGE and blotted with anti-Rab5 antibody. For comparison, actin and the NMDA receptor (as a cellular membrane marker) are also shown; the enrichment of endosomes (Rab5 enrichment) was approximately 9.6-fold, on average ( \pm 3.9 , four experiments). (B) Synaptobrevin interacts with the early endosomal SNAREs. PNS from PC12 cells was centrifuged in a sucrose density gradient, a band highly enriched in early endosomes was isolated, and after solubilization with Triton X-100 immunoprecipitation was performed with a monoclonal antibody against synaptobrevin (left lane); controls are shown in the second lane (no antibody added). The corresponding supernatants (Sup) are shown in the respective position (lanes 3-4). The interacting SNAREs were identified by immunoblot analysis. A total of $10 \%$ of the total sample is loaded in every lane. Typical blots of three independent experiments are shown. (C) Quantification by densitometry of coprecipitation, from the blots presented in panel B; the band intensities were normalized to starting material (see Materials and methods). The 'no antibody' value corresponds to the mean value of all negative controls presented. (D) Other SNARE interactions in the early endosomal fraction. Immunoprecipitations were performed with antibodies against the SNAREs indicated at the top of the figure. All immunoprecipitates were analyzed by immunoblotting for the SNAREs indicated. The 'no ab' value corresponds to the negative control, in which no antibody was added for immunoprecipitation. A total of $10 \%$ of the total sample is loaded in every lane. Boxed bands indicate immunoprecipitation of the blotted protein with its own antibody. Empty arrowheads indicate cognate interactions; full arrowheads indicate non-cognate interactions. Note that SNAP-25 was omitted from the analysis because none of the available antibodies detects SNAP-25 in assembled SNARE complexes. Immunoprecipitation of syntaxin 13 by vtila and syntaxin 1 is presented from PNS material; identical results were obtained with the endosomal fraction (data not shown). See Supplementary Table 1 for the immunoprecipitation efficiencies of all antibodies. 
in the precipitates, and no strong preference for the cognate SNAREs was observable. The R-SNARE VAMP4 does not precipitate with synaptobrevin, which is to be expected if the cosedimenting SNAREs represent bona-fide four helix bundles with a QabcR composition (see also below).

Next, we immunoprecipitated VAMP4, syntaxin 13, syntaxin 6 , vtila and syntaxin 1 and analyzed the precipitates for the presence of the other SNAREs (SNAP-25 was omitted because none of a battery of antibodies were capable of precipitating SNAP-25 in complex with other SNAREs). Representative blots are shown in Figure 2D. As for synaptobrevin, no preference for cognate SNAREs was detectable. For instance, traces of SNAP-25 were detected in the precipitates of VAMP4, syntaxin 13 and syntaxin 6 (non-cognate). Conversely, interactions were seen between syntaxin 6 and VAMP4, vtila and syntaxin 6, and syntaxin 1 and SNAP-25, that all are considered to represent cognate SNARE complexes.

Do the co-precipitating SNAREs represent genuine SNARE complexes or nonspecific associations mediated, for instance, by the transmembrane domains? SNARE complexes are disassembled by the AAA-ATPase NSF that is abundantly present in the cytosol. Therefore, we incubated post-nuclear supernatants (PNSs), spiked with rat brain cytosol, to provide additional NSF, in the presence and absence of ATP, before solubilization and immunoprecipitation. Figure 3 shows the results for syntaxin 13 , an early endosomal SNARE, for which relatively strong non-cognate interactions were observed (see Supplementary Figure 2 for corresponding results obtained for syntaxin 6). Essentially, no coprecipitating bands are detected in the presence of ATP, whereas in the absence of ATP, interactions between syntaxin 13 and synaptobrevin are evident (Figure 3A, arrowhead). We quantified the immunoprecipitation results obtained in three independent experiments (Figure 3B). Interestingly, syntaxin 13 is associated more strongly with synaptobrevin than with its cognate SNARE partners. ATP preincubation eliminates all of the interactions, both cognate and non-cognate. To confirm that the ATP effects are due to the action of NSF, reactions were carried out in the presence of ATP and the NSF inhibitor $\mathrm{N}$-ethylmaleimide (NEM). Again, the interaction between synaptobrevin and syntaxin 13 was evident (Figure 3C), although the degree of coprecipitation was lower than in the case of ATP depletion (which has also been observed in other systems; Carr et al, 1999). Very similar results were obtained when a rat brain fraction enriched in synaptic vesicles was used (Supplementary Figure 3), which is known to contain full complements of early endosomal and neuronal SNAREs (Rizzoli et al, 2006). Finally, to ensure that the non-cognate interactions are not only observable in isolated organelles, immunoprecipitations were carried out with intact PC12 cells as starting material, which were preincubated in the presence or absence of NEM as an NSF inhibitor. Again, non-cognate complexes were precipitated when NSF was inhibited (Supplementary Figure 3).

We conclude that the SNARE complexes isolated by immunoprecipitation represent genuine SNARE complexes that involve SNARE motifs and that are sensitive to NSF-driven disassembly. They represent cis-complexes, as they appear in conditions where no fusion is observed-in the presence of NEM or the absence of ATP (Supplementary Figure 1; Brandhorst et al, 2006). These complexes are formed in intact
A
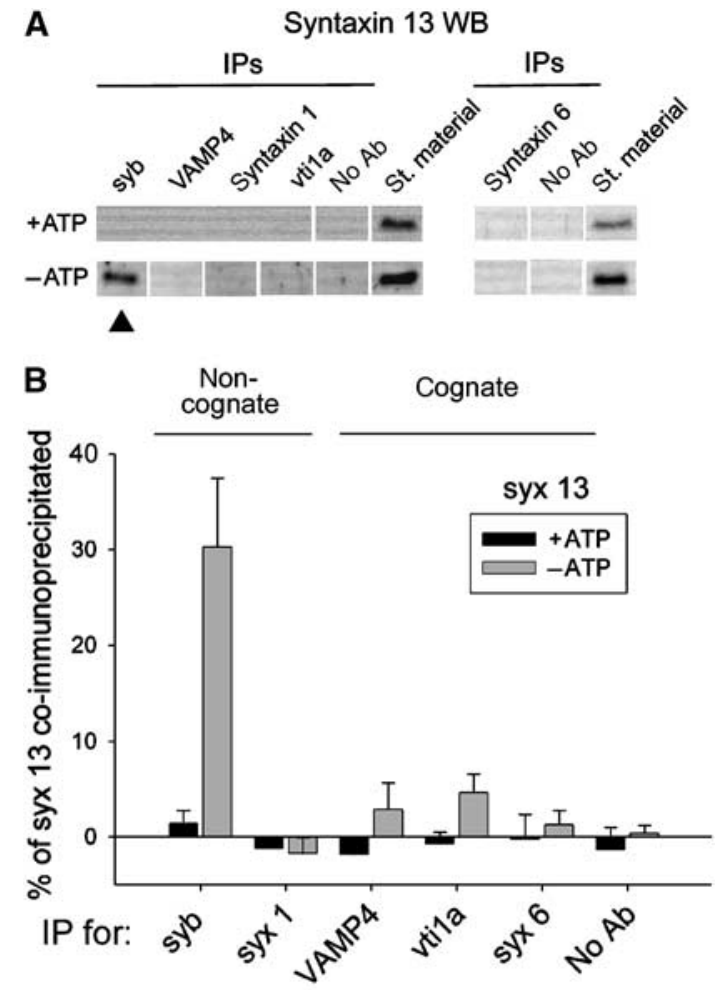

C

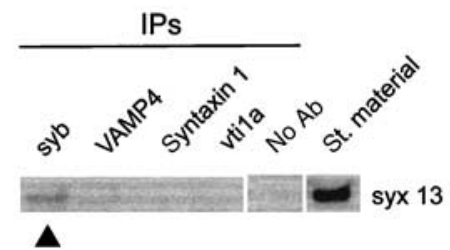

Figure 3 The non-cognate SNARE interactions represent genuine SNARE complexes in endosomal membranes. (A) PNSs were incubated with rat brain cytosol in presence or absence of ATP, and immunoprecipitations were performed after detergent extraction. Typical immunoblots for syntaxin 13 are shown. Note the presence of bands in the synaptobrevin precipitants in absence, but not in presence of ATP (arrowheads). (B) Quantification of syntaxin 13 coimmunoprecipitation. Bands were quantified by densitometry (see Materials and methods); averages \pm s.e.m. from three independent experiments are shown. (C) The interaction between synaptobrevin and syntaxin 13 is detectable in absence of NSF activity (arrowhead). PNS fractions were incubated in presence of ATP, cytosol and NEM, to block NSF activity.

membranes, and not after membrane solubilization, because they are eliminated if the NSF-mediated disassembly is allowed to proceed on intact membranes (whereas NSF activity was always inhibited in our solubilization and immunoprecipitation conditions).

\section{The relative amounts of cognate and non-cognate SNARE complexes depend on the relative concentrations of SNAREs}

Table I summarizes, in a qualitative manner, all of the interactions that were observed in the different preparations. It is evident that (i) not all interactions are observed in all preparations, with the complexes of synaptobrevin being the most consistent, and (ii) that in most cases, coprecipitation is observable only upon precipitation of one of the interacting partners, but not vice versa. 


\begin{tabular}{|c|c|c|c|c|c|c|c|}
\hline \multirow[t]{2}{*}{ EE } & PNS LS1 & \multicolumn{6}{|c|}{ Immunoprecipitated proteins } \\
\hline & & $\begin{array}{c}\text { Syntaxin } \\
1\end{array}$ & Syb & $\begin{array}{c}\text { Syntaxin } \\
13 \\
\end{array}$ & $\begin{array}{c}\text { Syntaxin } \\
6 \\
\end{array}$ & Vti1a & VAMP4 \\
\hline \multirow{7}{*}{ 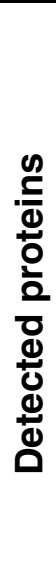 } & $\begin{array}{c}\text { SNAP } \\
25\end{array}$ & & & & & & 0 \\
\hline & $\begin{array}{c}\text { Syntaxin } \\
1\end{array}$ & & & & & & \\
\hline & Syb & & & & & & \\
\hline & $\begin{array}{c}\text { Syntaxin } \\
13\end{array}$ & & & & & & \\
\hline & $\begin{array}{c}\text { Syntaxin } \\
6\end{array}$ & & & & & & \\
\hline & Vti1a & & & & & & \\
\hline & VAMP4 & & & & & & \\
\hline
\end{tabular}

PNS, post-nuclear supernatant.

The blue circle indicates interactions observed in the early endosomal fraction from PNS of PC12 cells, the green the ones observed in PNS and the red circle the ones observed in the LS1 fraction from rat brain. The cognate interactions are enclosed by the cyan borders.

To explain these seemingly discrepant results, it needs to be borne in mind that the individual SNAREs are not present in stoichiometric amounts. For instance, if synaptobrevin is 50 times more abundant than vtila, and quantitative precipitation of synaptobrevin brings down $\sim 15 \%$ of the vtila pool (Figure 2C), it is evident that quantitative immunoprecipitation of vtila would result in the cosedimentation of only $\sim 0.3 \%$ of the synaptobrevin present in the starting extract, which would be difficult to discriminate from nonspecific binding to the beads. To clarify this issue, we determined the absolute amounts of the SNAREs in purified early endosomes and, for comparison, in both PC12 cell-derived PNS and a synaptic vesicle-enriched fraction isolated from rat brain synaptosomes, using purified recombinant proteins as standards (Table II; Supplementary Table 2). From the data it is evident that synaptobrevin is much more abundant than any of the other immunoprecipitated SNAREs, explaining whyrelative to the starting material-no significant amounts of synaptobrevin are detectable in the immunoprecipitates of most SNAREs, whereas, conversely, all proteins except the R-SNARE VAMP4 are detectable in the immunoprecipitates of synaptobrevin. Furthermore, there are major differences between the different preparations. Syntaxin 1 is 2 - to 3 -fold more abundant in the synaptic vesicle-enriched fraction LS1 than in PNS or early endosomes, which explains why the cognate partner synaptobrevin is only observable in precipitates derived from LS1; the same can be stated for the non-cognate syntaxin 6 . The level of VAMP4 is about an order of magnitude lower in LS1 than in early endosomes or PNS-thus, it is not detectable as interaction partner in LS1.

Thus it appears that the formation of cis-complexes is, at least in part, is governed by mass action. To substantiate these conclusions, we generated a simple Monte Carlo model describing SNARE complex formation between the two
Table II Quantification of the immunoprecipitated proteins in each preparation

\begin{tabular}{lccc}
\hline$p M$ & LS1 & Early endosomes & PNS \\
\hline Syntaxin 13 & $0.58 \pm 0.06$ & $3.32 \pm 0.4$ & $1.29 \pm 0.32$ \\
Syntaxin 6 & $0.46 \pm 0.07$ & $5.44 \pm 0.19$ & $2.59 \pm 0.09$ \\
Vti1a & $0.18 \pm 0.03$ & $0.68 \pm 0.16$ & $0.26 \pm 0.02$ \\
Vamp4 & $0.015 \pm 0.005$ & $0.43 \pm 0.03$ & $0.28 \pm 0.04$ \\
Syntaxin 1 & $7.44 \pm 0.87$ & $2.94 \pm 0.55$ & $2.01 \pm 0.32$ \\
Synaptobrevin & $127 \pm 9.2$ & $37.89 \pm 1.65$ & $6.77 \pm 2.99$ \\
\hline
\end{tabular}

PNS, post-nuclear supernatant.

Standard curves of purified recombinant proteins were separated by SDS-PAGE alongside with different amounts of PNSs, early endosomal and LS1 fractions, followed by immunoblot analysis. The protein amounts in each preparation were calculated by interpolation. Means \pm s.e.m. from 3-5 independent measurements are shown. The results are presented as molar ratios in Supplementary Table 2 .

R-SNAREs synaptobrevin and VAMP4, and acceptor sites formed by the endosomal Q-SNAREs (syntaxin 13/vtila/syntaxin 6) or by the exocytotic Q-SNAREs (syntaxin 1/SNAP-25). All SNAREs were inserted in their correct stoichiometric proportions (see Supplementary Table 2): VAMP4 (five copies), early endosomal Q-SNARE complex (eight, limited by the low copy number of vtila), synaptobrevin (439) and the exocytotic QSNARE complex (34). The density of the SNAREs per unit of surface (Figure 4A) was adjusted using synaptic vesicles as model for which such quantitative data are available (Takamori et al, 2006). For simplicity, we made the assumption that the acceptor sites are permanently stable.

The R-SNAREs were allowed to interact freely and form complexes with the QaQbQc acceptors. Collision of the cognate partners resulted always in SNARE complex formation. To test the non-cognate behavior, we varied this 
A

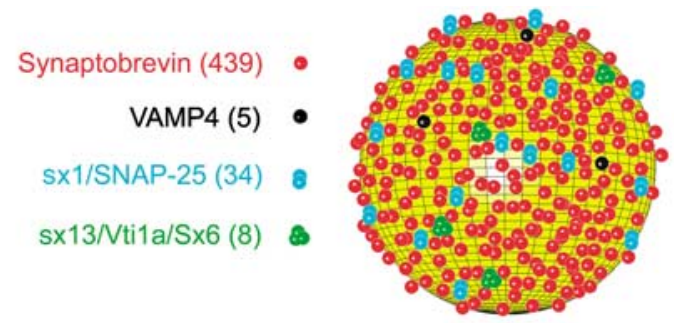

B
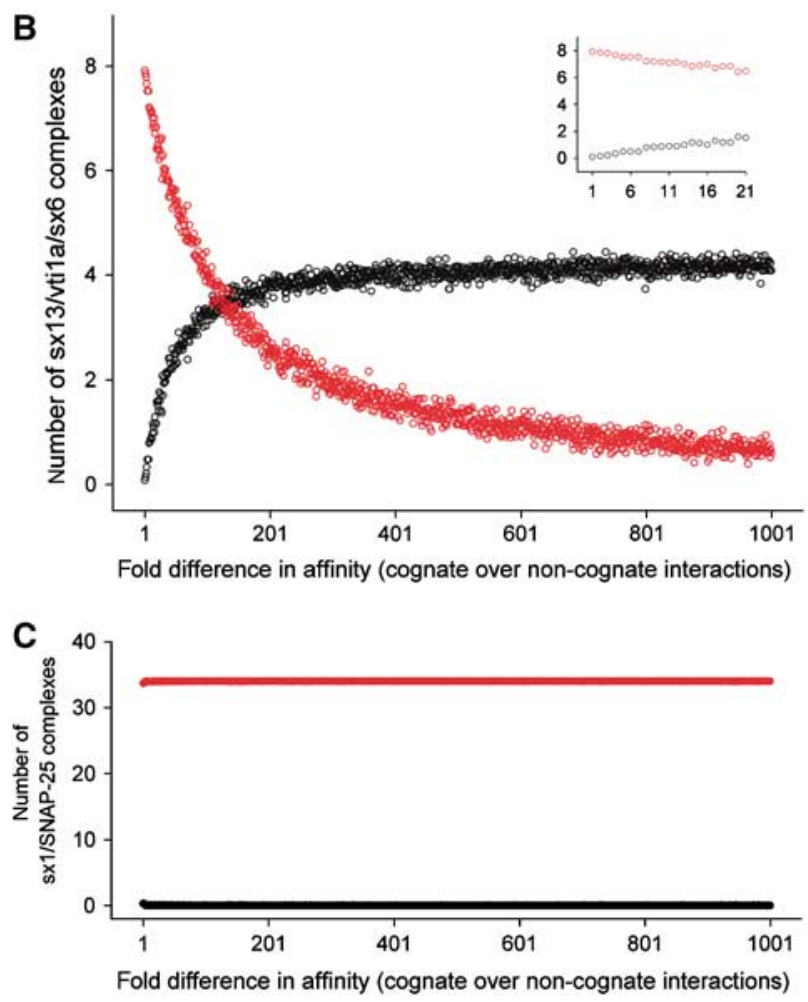

Figure 4 Mass action determines cis-complex formation. We simulated the behavior of SNAREs in a model endosome, containing synaptobrevin, VAMP4, the early endosomal Q-SNARE acceptor complex (syntaxin 13/vtila/syntaxin 6) and the exocytotic QSNARE acceptor complex (syntaxin 1/SNAP-25). We allowed the SNAREs to mix and interact for 1000 iterations, and then counted the number of both cognate and non-cognate complexes. We varied the affinity of R-SNAREs for their non-cognate complex acceptors between 1 and $1 / 1000$. The affinity for the cognate complex was always 1 . The fold difference in affinity is indicated on the $x$-axis. (A) The levels of the different elements are shown in brackets; the graphic description of the model is provided to give an impression of the different SNARE densities. (B) The number of complexes containing the early endosomal Q-SNARE acceptor (synaptobrevincontaining complexes, red; VAMP4-containing complexes, black). As synaptobrevin is the non-cognate partner here, its affinity decreases from left to right. The inset zooms on the first 20 points of the curves. Note that the number of synaptobrevin-containing complexes largely outnumbers that of VAMP4-containing complexes when the affinity difference is of $1-50$ fold. Some non-cognate complexes form even at 1000 -fold differences in affinity, when VAMP4 is still limited to only 4 of the 5 possible complexes (see also Supplementary Figure 4). (C) The number of complexes containing the exocytotic Q-SNARE acceptor. As VAMP4 is the non-cognate partner here, its affinity decreases from left to right. Note that, due to the differences in concentration, essentially no VAMP4-containing (non-cognate) complexes form, even when the affinity is 1 for both the R-SNAREs (the left-most point).

parameter (the probability of complex formation per collision) between 1 and 1:1000; this is equal to saying that we varied the fold difference in affinity for formation of cognate and non-cognate complexes between 1 and 1:1000. Newly formed QaQbQcR complexes persisted throughout the simulation, mimicking an 'NSF inhibition' situation. The model predicted fairly accurately our in vitro co-immunoprecipitation results (see the Materials and methods section for the in silico/in vitro comparison). Synaptobrevin out-competed VAMP4 when their probabilities to form complexes were comparable, until VAMP4 binds more than 150-fold more efficiently. Moreover, as the number of QaQbQc acceptor sites is higher than the number of VAMP4 molecules, some synaptobrevin-containing complexes will be able to form even when the synaptobrevin affinity is infinitesimally small (Figure 4B). Finally, VAMP4 forms only minor amounts of non-cognate complexes with syx 1/SNAP-25, even when we assume it can bind to them perfectly well (Figure 4C), showing that SNARE concentrations also determine which complexes cannot form.

Thus, our data support the view that the relative amounts of different SNARE complexes in a given membrane are primarily dependent on the stoichiometric ratio between these SNAREs. In the early endosomes of PC12 cells, these proportions predict a high degree of non-cognate complexes. Surprisingly, this is also true if one assumes that there is a strong intrinsic preference for cognate SNARE pairing in the 'cis-'configuration.

\section{Addition of soluble SNAREs onto native membranes reveals preference but not absolute specificity for cognate SNARE pairing}

The data described so far show that intact membranes do not contain proof-reading mechanisms selecting cognate SNAREs and/or preventing non-cognate SNAREs from forming complexes, at least not to the degree required to explain the SNARE specificity of endosome fusion and exocytosis, respectively. This finding is highly surprising, not only because it challenges co-immunoprecipitation as a means for defining cognate SNARE complexes, but also because it shows that SNARE proof-reading mechanisms must exist for an upcoming fusion event, which only operate when the SNAREs are in trans-configuration.

To assess whether there are indeed membrane-intrinsic proof-reading mechanisms for SNARE pairing, we asked whether there is preference for cognate SNAREs when soluble SNARE partners are added from the outside. This approach allows for probing the capacity of membrane-resident SNAREs to form complexes with cognate and non-cognate SNAREs at defined concentrations and in the absence of docking complexes that may form before membrane fusion.

We used inverted lawns of PC12 cells, which contain endogenous syntaxin 1 and SNAP-25. We have shown previously that these SNAREs are active and can be driven into SNARE complexes by the addition of recombinant synaptobrevin (Lang et al, 2002). We performed competition experiments to see whether the endogenous exocytotic SNAREs have a preference for their cognate SNARE partners. Fluorescently labeled syntaxin 1 was added to the plasma membrane sheets, resulting in binding (Figure $5 \mathrm{~A}$ ) that is specific, as it is abolished by removing SNAP-25 and is reverted by NSF (Lang et al, 2002). When excess unlabeled Qa SNAREs (syntaxin 1, syntaxin 13, or the late endosomal syntaxin 7) were added at identical concentrations $(10 \mu \mathrm{M})$, only syntaxin 1 , but neither syntaxin 13 nor syntaxin 7 , was 
able to compete effectively with the binding of labeled syntaxin 1 (Figure 5B). Similarly, the late endosomal RSNARE endobrevin/VAMP8 was less efficient than synaptobrevin in competing with the binding of labeled synaptobrevin (Figure 5C). Endobrevin was used instead of VAMP4 because the VAMP4 fragment lacking the transmembrane domain tends to precipitate.

These data clearly show that in intact membranes the neuronal Q-SNAREs SNAP-25 and syntaxin discriminate between externally added synaptobrevin and endobrevin, although no such discrimination is observed when the proteins are combined in solution or when they are used in liposome fusion experiments. However, do these findings imply that non-cognate SNARE complexes cannot form at all under these conditions? Two types of experiments were performed to address this issue.

First, we asked whether externally added endobrevin is capable of forming complexes with endogenous syntaxin

A
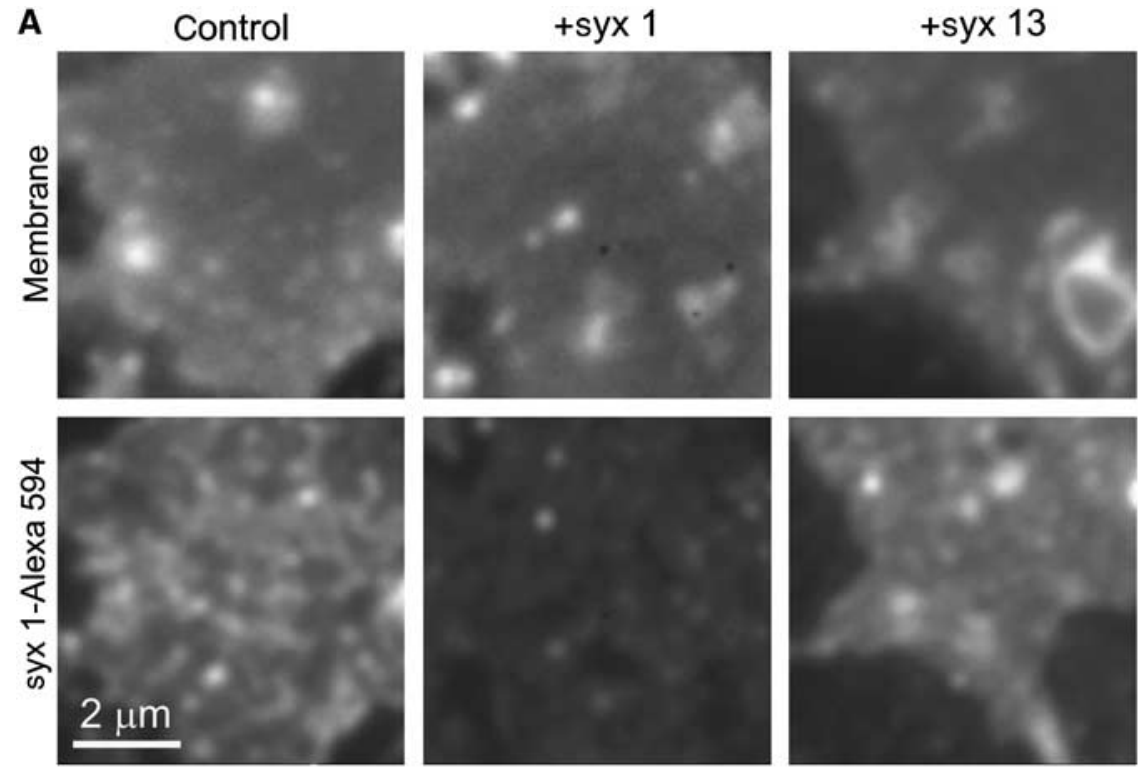

B
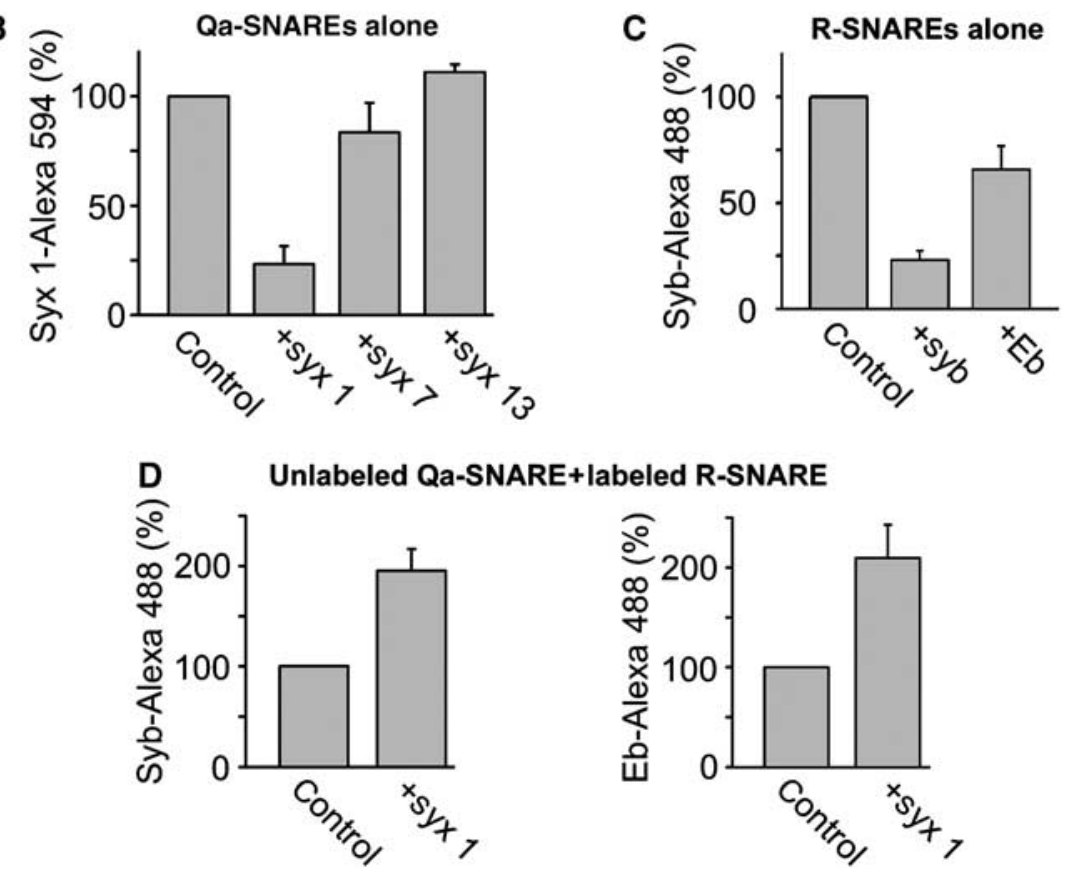

Figure 5 Exocytotic SNAREs bind relatively specifically to plasma membrane sheets. (A) Membrane sheets were treated with $1 \mu \mathrm{M}$ syntaxin 1A-Alexa 594 alone (left), or in the presence of excess $(10 \mu \mathrm{M})$ syntaxin 1 (middle) or syntaxin 13 (right). The top three panels show the plasma membranes (by staining with the lipid tracer TMA-DPH). Alexa 594 incorporation in the membrane is shown in the bottom panels. (B) Quantification of syntaxin 1A-Alexa 594 binding; 10-fold excess unlabeled competing SNAREs were added as indicated. (C) Similar experiments were performed to investigate synaptobrevin binding to membrane sheets. Unlabeled synaptobrevin or endobrevin were added as 10-fold excess, as indicated. (D) Membrane sheets were reacted with Alexa 488-labeled R-SNAREs, either alone or in the presence of equimolar amounts $(4 \mu \mathrm{M})$ of syntaxin 1 . The graph on the left shows the results for synaptobrevin; the graph on the right indicates the results for endobrevin. 
1/SNAP-25. To test this, we monitored the binding of synaptobrevin and endobrevin in presence of unlabeled syntaxin 1. Since the plasma membrane has a high excess of SNAP-25, syntaxin 1 addition results in formation of syntaxin:SNAP-25 complexes that in turn serve as additional binding sites for synaptobrevin (Figure 5D; Lang et al, 2002). When binding of endobrevin was monitored instead, a similar two-fold increase in binding was observed upon syntaxin 1 addition. This finding, together with the data shown in Figure 5C, shows that endobrevin does form SNARE complexes with endogenous syntaxin 1/SNAP-25, although less well than synaptobrevin.

Second, we investigated if soluble R-SNAREs, added alone in high concentrations, are capable of overcoming the selectivity filter and drive both cognate and non-cognate QSNAREs into SNARE complexes. We incubated PC12 cellderived PNSs with saturating amounts $(25 \mu \mathrm{M})$ of myc-tagged variants of synaptobrevin, VAMP4 and endobrevin, and analyzed their ability to immunoprecipitate endogenous membrane-resident SNAREs (note that recombinant mycVAMP4 is more stable than the untagged protein). We used myc-tagged variants and immunoprecipitation with anti-myc antibodies to differentiate the exogenous SNAREs from their endogenous counterparts. To dissociate all pre-existing endogenous complexes, we first incubated the organelles in presence of ATP and then inhibited NSF by NEM to prevent disassembly of the newly formed complexes. As shown in Figure 6, the three R-SNAREs pulled down both cognate and non-cognate SNAREs to a similar extent.

We conclude that intact membranes contain a proof-reading mechanism for SNARE pairing that appears to preferably operate in 'trans'. However, the discrimination between cognate and non-cognate SNAREs as observed under these conditions does not suffice to explain the absolute selectivity in fusion, particularly when considering that in homotypic endosome fusion in PC12 cells, the membranes contain much more synaptobrevin than VAMP4.

\section{Lateral segregation of SNAREs at contact/docking sites contributes to trans-SNARE pairing specificity}

In general, membrane fusion between intracellular organelles is initiated by the formation of tethering/docking complexes that include proteins interacting with SNAREs. Thus it is conceivable that during docking, cognate SNAREs are included within such docking complexes whereas non-cognate SNAREs are excluded. While the resolution of the light microscope does not suffice to discern such subdomains in native endosomes, we took advantage of the large endosomes generated in Rab5-Q79L-overexpressing cells (Figure 1). Occasionally, pairs of closely apposed endosomes are detectable (Figure 7A). The fate of each pair of such endosomes is difficult to predict, but in general they likely represent prefusion intermediates, as constricted endosomes indicative of recent fusion of large organelles are seen relatively frequently (Figure 7D). While large contact sites are infrequent, we succeeded in performing a number of immunostainings for synaptobrevin on such sites (three, in three independent experiments; depicted in Figure 7A-C). In all cases, it was evident that synaptobrevin appeared to be excluded from the contact sites. In contrast, comparatively more syntaxin 6 than synaptobrevin was found at one interface that was immunostained for both of the molecules (Figure 7C; see also
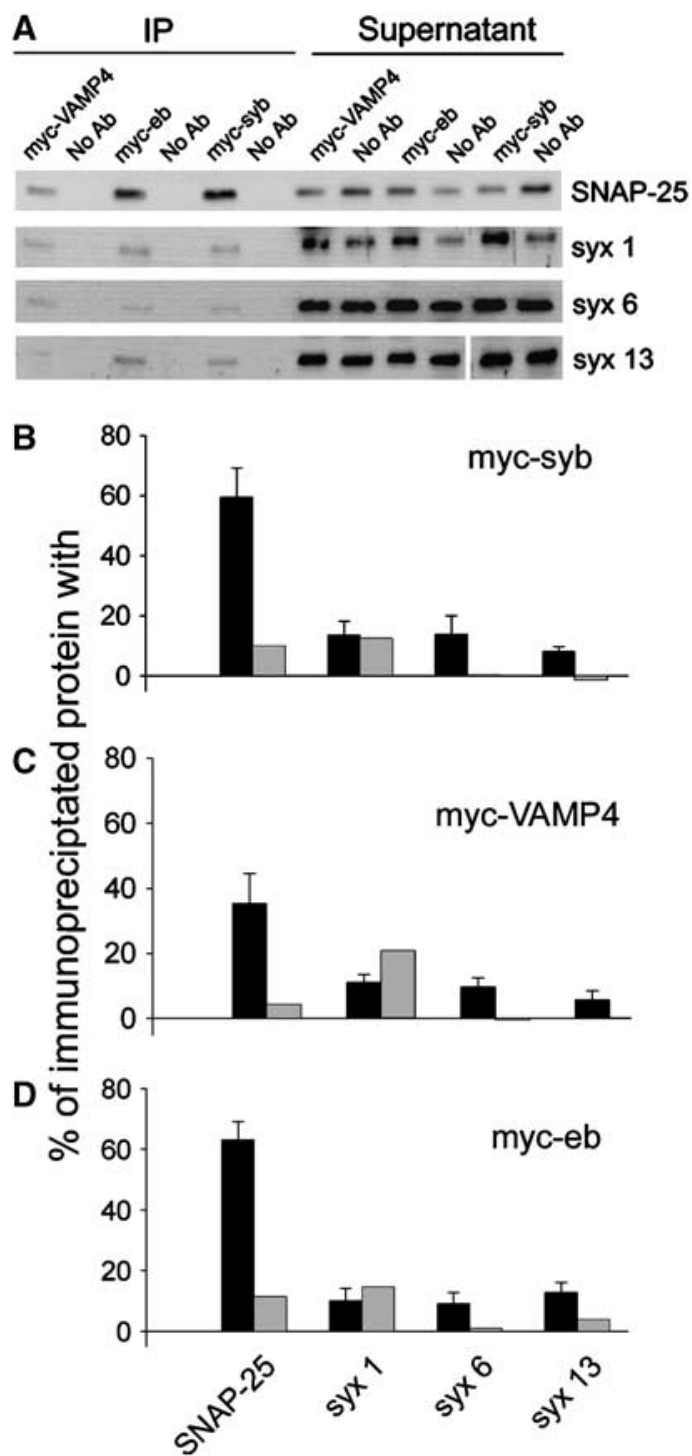

Figure 6 Promiscuous SNARE complexes form between membrane-resident SNAREs and SNAREs added externally at high concentrations. PNS of PC12 cells was incubated with $25 \mu \mathrm{M}$ of myctagged proteins synaptobrevin, VAMP4 and endobrevin, respectively, in presence of ATP at $37^{\circ} \mathrm{C}$. After $45 \mathrm{~min}$ of incubation, $1 \mathrm{mM}$ NEM was added to block disassembly and incubation continued for $30 \mathrm{~min}$. Reactions were centrifuged for $25 \mathrm{~min}$ at $300000 \mathrm{~g}$, to eliminate the soluble SNAREs molecules not bound to the membranes. Pellets were resuspended in extraction buffer and immunoprecipitated with anti-myc antibodies. Each immunoprecipitation was accompanied by a negative control in which no antibody was used. The precipitants were analyzed by SDS-PAGE Western blotting. We present the results for four SNAREs for which co-immunoprecipitation bands were observed: SNAP-25, syntaxin 1 , syntaxin 6 and syntaxin 13. (A) Typical blots are presented. (B-D) The amounts of protein coprecipitating with myc-synaptobrevin (B), myc-VAMP4 (C) and myc-endobrevin (D) were quantified; averages \pm s.e.m. from four independent experiments are shown (black bars). Gray bars indicate the amount of SNARE complexes that formed after solubilization (see Materials and methods for details). Three independent experiments were performed in conditions identical to those of the experiments in panels A-D; bars show average.

Supplementary Figure 5). Thus, in sharp contrast to the overlapping distribution in other parts of the endosome membrane (Figure 1), it appears that lateral segregation occurs at sites where membranes attach, before fusion. 

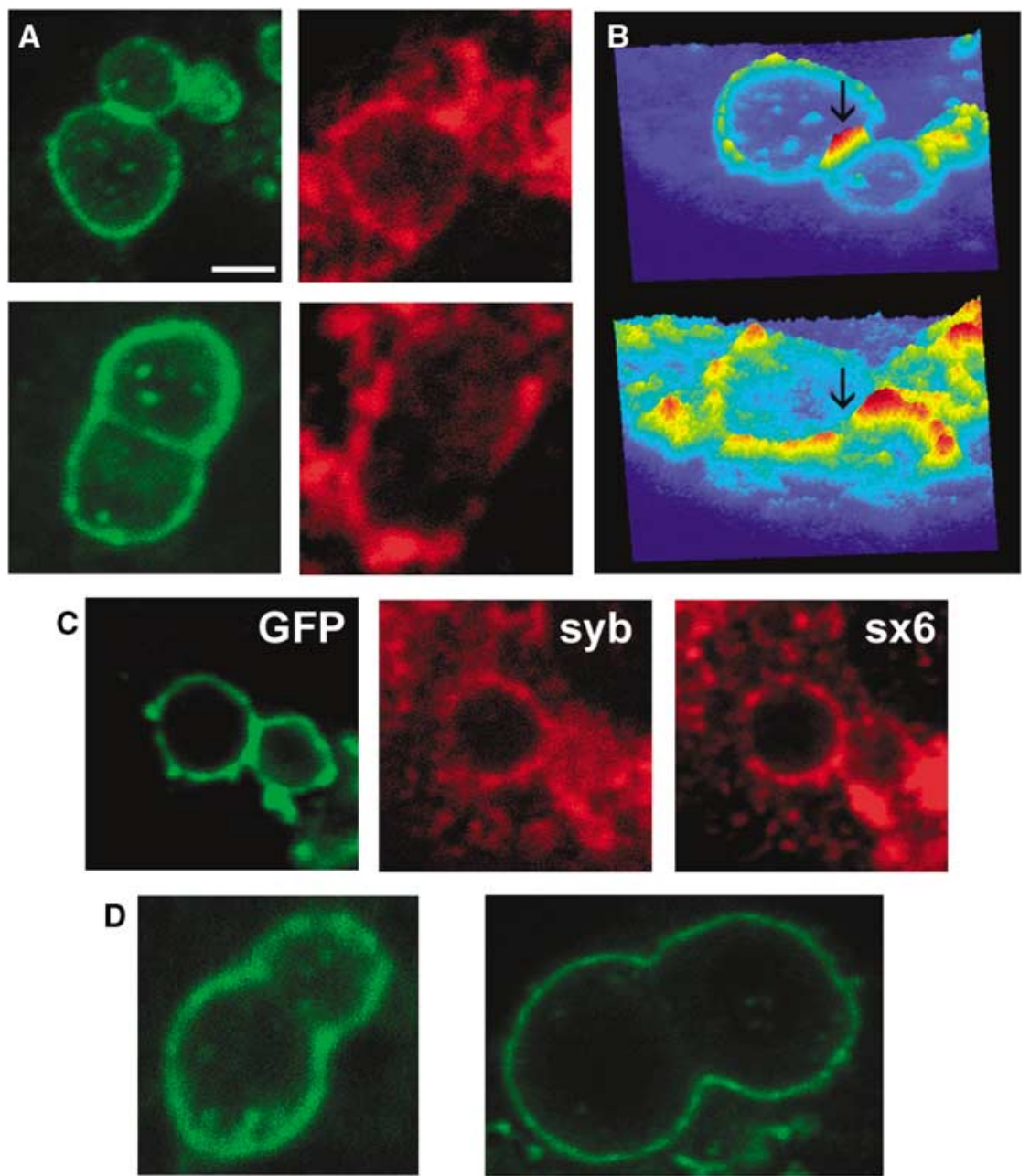

Figure 7 Synaptobrevin appears to avoid the interfaces between endosomes. PC12 cells were transfected with a plasmid expressing GFP-Rab5Q79L and immunostained for synaptobrevin as in Figure 1. (A) Two endosome pairs showing clear interfaces; green, Rab5-GFP; red, synaptobrevin. Scale bar, $1 \mu \mathrm{m}$. (B) Three-dimensional view of the endosomes in the top panels of A. Top, Rab5-GFP. Bottom, synaptobrevin. Arrows point to the interface. (C) Endosome pair similarly stained for synaptobrevin (middle) and syntaxin 6 (right). (D) The fate of the endosomes pressing against each other is difficult to predict; they most likely fuse, as we see relatively abundant constricted (i.e. post-fusion) structures.

Non-cognate SNAREs appear to be excluded, which contributes to SNARE specificity.

In conclusion, two factors that are probably independent from each other and that operate in concert appear to be responsible for the selectivity of trans-SNARE pairing. First, there appears to be a preference for cognate SNAREs in the trans-configuration, and second, lateral segregation leads to a local enrichment of cognate and/or de-enrichment of noncognate SNAREs, together greatly increasing the likelihood for the formation of cognate trans-complexes despite an excess of non-cognate SNAREs in the endosome membrane. To test whether specificity can indeed be achieved with these two mechanisms, we again performed Monte Carlo simulations (Figure 8). We modeled four different situations; in all of them we assumed that a 10 -fold preference for cognate SNAREs is seen upon trans-complex formation, and that trans-complexes, once formed, do not dissociate anymore. First (model I), we assumed that both exocytotic and endosomal SNAREs are randomly distributed. In a second model (II) we assumed that the concentration of the exocytotic SNAREs at the contact site is reduced by a factor of 10 -fold. Third (III), we assumed that the concentration of early endosomal SNAREs is increased by three-fold at the contact site. Finally (IV), we coupled the reduction in exocytotic SNAREs with the increase in endosomal SNAREs. The proportion of cognate versus non-cognate complexes increases steadily from I to IV (Figure 8A). If we assume that four complexes are required for the fusion event, it is clear from the simulation that specificity alone (model I) is not sufficient for fusion (Figure 8B), but that coupling specificity with a mild enrichment/de-enrichment of the appropriate SNAREs results in fusion on every run (model IV).

\section{Discussion}

In the present study, we have investigated at which step within the SNARE assembly-disassembly cycle pairing specificity is achieved. Using SNAREs that are specialized for two distinct fusion steps, but strongly overlap in their recycling pathways and interact nonspecifically in vitro, we found that they are capable of forming nonspecific cis-complexes while being neighbors in the same membranes, but exhibit preference for cognate SNAREs added from the outside. Furthermore, we observed lateral SNARE segregation at 

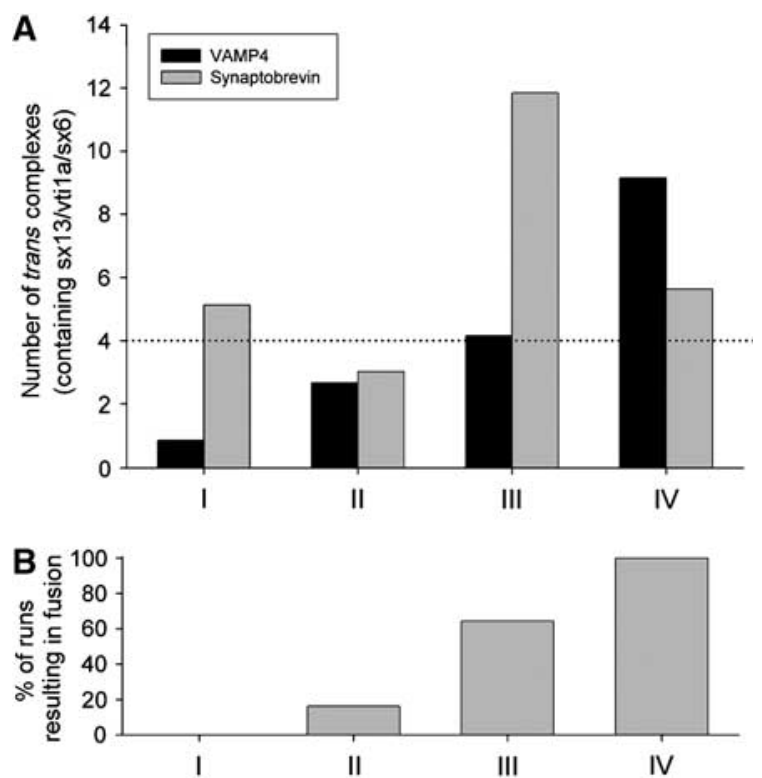

Figure 8 Fusion specificity is achieved by synergistically operating mechanisms. We simulated the behavior of two interacting endosomes of $\sim 233$-nm diameter, each containing 25 VAMP4 molecules, 40 early endosomal Q-SNARE acceptor complexes (syntaxin 13/ vti1a/syntaxin 6), 2195 synaptobrevin molecules and 170 exocytotic Q-SNARE acceptor complexes (syntaxin 1/SNAP-25). We allowed the SNAREs from the two endosomes to interact in the interface between them for 1000 iterations. All trans-complexes that formed remained stable until the end of simulation; cis-complexes formed as well, but were always disengaged before the start of the next iteration. (A) We monitored the number of trans-complexes containing the early endosomal Q-SNAREs (with either VAMP4, shown in black, or synaptobrevin, shown in gray). A 10 -fold preference for cognate complex formation was modeled (see Supplementary Figure 6 for other preference values). Four models are presented as follows: I, random distribution of both exocytotic and endosomal SNAREs; II, 10-fold de-enrichment of exocytotic SNAREs from the interface; III, three-fold enrichment of endosomal SNAREs in the interface and IV, both a de-enrichment of exocytotic SNAREs and an enrichment of the endosomal ones. (B) Formation of at least four VAMP4-containing complexes was required to consider the two endosomes as 'fused'. The average percentage of fused pairs for $30-45$ simulations is shown.

sites where endosomes contact each other and which may represent prospective fusion sites. We conclude that pairing specificity is achieved by two cooperating mechanisms, a selectivity filter that appears to operate preferably in transconfiguration, and lateral segregation that is probably achieved after vesicle docking.

Co-immunoprecipitation of SNAREs from detergent extracts, occasionally in combination with pull-down experiments using immobilized SNAREs, is being widely used as a tool to identify cognate SNARE complexes (see for example Prekeris et al, 1998; Zhang and Hong, 2001; Sun et al, 2003). Our results now show that considerable caution needs to be employed in interpreting co-precipitation data. We conclude that (i) the coprecipitation of SNAREs, even if seemingly specific, cannot be used as evidence for their functional interaction in a fusion reaction, and (ii) conversely, the inability to detect coprecipitation does not suffice to rule out that SNAREs may cooperate in fusion. In view of these conclusions, our findings explain why there have been so many discrepancies in the assignment of SNAREs to homotypic fusion of early endosomes, particularly with respect to the involvement of the neuronal SNAREs synaptobrevin and SNAP-25 (Prekeris et al, 1998; Hirling et al, 2000; Sun et al, 2003; Aikawa et al, 2006). Co-precipitation may be safely used to assume that the particular SNAREs are able to interact in the same membrane (as long as controls are performed to exclude complex formation after membrane solubilization). This may not be completely irrelevant, as non-cognate complexes may actually fine-tune the fusion machinery by regulating the nature and the levels of SNAREs available for trans-complex formation (Varlamov et al, 2004).

Our data shed new light on the controversially discussed question of how specificity of SNARE pairing is achieved. Although only tested on two sets of SNAREs, several conclusions can be made: first, SNAREs residing in the same membrane form both cognate and non-cognate complexes. While such cis-complexes probably never accumulate appreciably in a healthy cell containing NSF and high ATP-levels, they show that in cis-configuration there is very little (if any) pairing specificity. Second, our simulations show that due to the overwhelming abundance of synaptobrevin, non-cognate complexes are expected to dominate even if there were a 100fold preference for cognate interactions, cis-interactions being thus condemned to non-cognate complex formation, virtually irrespective of SNARE preference. Third, a preference for cognate SNAREs is indeed observable when SNAREs are added from the outside.

What is the nature of the selectivity filter? Several lines of evidence suggest that SM proteins may play a major role. In yeast, the SM protein Sly1p was shown to enhance pairing specificity of yeast SNAREs in vitro (Peng and Gallwitz, 2002). Similarly, the neuronal SM protein Munc-18 appears to increase pairing specificity in liposome fusion experiments (Shen et al, 2007). In agreement with this concept, we have shown recently that synaptobrevin binding to syntaxin 1/SNAP-25 on plasma membrane lawns triggers a displacement of Munc-18, whereas endobrevin is much less efficient (Zilly et al, 2006). Although more evidence is needed, a picture is emerging, according to which SM proteins (perhaps in conjunction with other proteins such as the HOPS complex involved in the docking and activation of yeast vacuoles, Collins et al, 2005) stabilize a SNARE acceptor complex that is more specific for the final cognate SNARE(s) than if the SNAREs are allowed to associate randomly with each other. For the neuronal SNAREs, the nucleation site for R-SNARE binding has recently been shown to be located at the membrane-distal N-terminal end of the SNARE motif (Pobbati et al, 2006). It is conceivable that an R-SNARE residing in cis-configuration is sterically disadvantaged to react with such an acceptor complex, whereas no such hindrance is encountered when the complex is approached 'from the top' in trans-configuration.

A major conclusion of our work is that a strong preference for cognate pairing in trans, of even 100-fold, is not sufficient for fusion (Supplementary Figure 6). Thus, a second mechanism needs to be called, which is provided by lateral segregation of SNAREs. Recruitment of cognate SNAREs to the prospective fusion site while excluding non-cognate SNAREs obviously improves the chances to fuse. This is in line with the findings that yeast vacuole SNAREs do tend to enrich at the organelle interfaces (Wang et al, 2003; Fratti et al, 2004). It is yet unclear how SNAREs actually enrich at 
different sites; however, a number of proteins interact with and may recruit SNARE molecules, such as HOPS and the Rab5-dependent docking machinery (see review by Jahn and Scheller, 2006).

Although the molecular details of both mechanisms (SNARE specificity and SNARE segregation) need to be further clarified, they provide yet another example for the principle of coincidence for achieving specificity: only the combination of two filters, each of which may only provide a moderate degree of selectivity, provides the specificity and robustness required for function.

\section{Materials and methods}

\section{Antibodies}

See Supplementary data for a complete list. All of the antibodies were previously described. Their immunoprecipitation efficiencies are shown in the Supplementary Table 1.

\section{Recombinant proteins}

The cytosolic fragment of endobrevin (residues 1-74) was subcloned into pET28a vector (Novagen, Madison, WI), which includes a thrombin cleavage site for the removal of the upstream $\mathrm{His}_{6}$-tag, using the primers gaggcacatatggaggccagtgggag and cgaattctacttcacattcttc caccag for PCR amplification. A cysteine mutant of endobrevin (S17C) was generated with the Quick Change site-directed mutagenesis kit (Stratagene) using the primers ggaacctgcagtgtgaggtggag and ctccacctcacactgcaggttcc and the cytosolic fragment of endobrevin (residues 1-74 in pET28a, see above) as template. All other constructs have been previously described (Supplementary data). Also, see Supplementary data for protein purification.

\section{Measurements of SNARE complex formation with \\ myc-tagged proteins after solubilization}

In the experiments, organelles were incubated with $25 \mu \mathrm{M}$ tagged SNAREs, before pelleting at $300000 \mathrm{~g}$, solubilization of the pellet and immunoprecipitation. We first quantified the amounts of myctagged proteins pelleting with the organelles. Quantification was performed by comparing the levels remaining on organelles with different amounts of purified protein, in Western blotting. We determined that $\sim 0.3-1 \mu \mathrm{M}$ SNAREs remained in the preparation at the time of solubilization. To control for SNARE complex formation during solubilization (Figure 7, gray bars), we added this amount of tagged SNAREs to pelleted organelles (which were identically incubated, but not in presence of myc-tagged SNAREs), then solubilized them and performed immunoprecipitations with anti-myc antibodies. The precipitants were analyzed by SDS-PAGE Western blotting against SNAP-25, syntaxin 1, syntaxin 6 and syntaxin 13. Bands were analyzed by densitometry; averages from three independent experiments ran in parallel are presented in Figure 7.

\section{References}

Aikawa Y, Lynch KL, Boswell KL, Martin TF (2006) A second SNARE role for exocytic SNAP25 in endosome fusion. Mol Biol Cell 17: 2113-2124

Antonin W, Riedel D, Fischer von Mollard G (2000) The SNARE Vtila-beta is localized to small synaptic vesicles and participates in a novel SNARE complex. J Neurosci 20: $5724-5732$

Brandhorst D, Zwilling D, Rizzoli SO, Lippert U, Lang T, Jahn R (2006) Homotypic fusion of early endosomes: SNAREs do not determine fusion specificity. Proc Natl Acad Sci USA 103: 2701-2706

Carr CM, Grote E, Munson M, Hughson FM, Novick PJ (1999) Sec1p binds to SNARE complexes and concentrates at sites of secretion. J Cell Biol 146: 333-344

Collins KM, Thorngren NL, Fratti RA, Wickner W (2005) Sec17p and HOPS, in distinct SNARE complexes, mediate SNARE complex disruption or assembly for fusion. EMBO J 24: $1775-1786$

\section{Enlarged-endosome image analysis}

This was performed as follows, using custom routines written in Matlab: images were first aligned using a least squares fit to a region of interest containing the endosome to be analyzed, and lines of interest were traced manually along the endosomal membrane. The intensity profiles in the cy 3 and cy 5 channels (representing two different SNARE proteins) were then compared by use of a correlation coefficient (as described, Brandhorst et al, 2006). As negative control we used the correlation between the intensity profile in the cy 3 channel and the intensity profile in the cy5 channel, placed in reverse order (mirrored). We used 1-pixel-wide lines of interest; enlarging the line of interest to 5 or 11 pixels did not result in significant differences (data not shown). Figure $1 \mathrm{E}$ is generated by rendering the endosome images into surfaces by use of the 'surface' routine in Matlab. The images were first smoothened by an averaging filter of 3 by 3 pixel size; accordingly, the line scans shown in Figure $1 \mathrm{~F}$ are filtered by use of a similar (3-pixel width) running average.

\section{Modeling}

To test the accuracy of the model in Figure 4, we compared the predicted immunoprecipitation rates with the experimental results from Figure 2C. When the difference between the probabilities of complex formation is only a few fold between cognate and noncognate complexes, the number of sx13/vtila/sx 6 complexes pulled down is $\sim 0.5$ for VAMP4 and $\sim 7$ for synaptobrevin (Figure $4 \mathrm{~B}$ ). This indicates that VAMP4 will pull down $\sim 1.3 \%$ of syntaxin 13 , $0.8 \%$ of syntaxin 6 and $\sim 6 \%$ of vtila (as they are present in copy numbers of 38,63 and 8 , respectively); this correlates perfectly with the fact that vtila was the only cognate SNARE for which we found measurable amounts in the VAMP4 precipitates (although only in the LS1 fraction; see Table I). Synaptobrevin is expected to immunoprecipitate $\sim 18 \%$ of syntaxin $13, \sim 11 \%$ of syntaxin 6 and $\sim 87 \%$ of vtila. The only large error $(\sim 6$-fold $)$ is for vtila (Figure 2C), which may be explained by an involvement of vtila isoforms (such as vtilb) in the non-cognate complexes in real endosomes.

All other procedures are described in the Supplementary data section.

\section{Supplementary data}

Supplementary data are available at The EMBO Journal Online (http://www.embojournal.org).

\section{Acknowledgements}

We thank Drs Daniel Zwilling, Tabez J Siddiqui and Wolfram Antonin for the gift of recombinant proteins. SOR is the recipient of a postdoctoral fellowship from the International Human Frontier Science Program Organization. This work was supported by a grant from the DFG to RJ (SFB 523, TP B6). We thank Dr Mikael Simons for the gift of Rab5-Q79L-GFP plasmid.
Fratti RA, Jun Y, Merz AJ, Margolis N, Wickner W (2004) Interdependent assembly of specific regulatory lipids and membrane fusion proteins into the vertex ring domain of docked vacuoles. J Cell Biol 167: 1087-1098

Hirling H, Steiner P, Chaperon C, Marsault R, Regazzi R, Catsicas S (2000) Syntaxin 13 is a developmentally regulated SNARE involved in neurite outgrowth and endosomal trafficking. Eur $J$ Neurosci 12: 1913-1923

Hong W (2005) SNAREs and traffic. Biochim Biophys Acta 1744: $120-144$

Humeau Y, Doussau F, Grant NJ, Poulain B (2000) How botulinum and tetanus neurotoxins block neurotransmitter release. Biochimie 82: 427-446

Jahn R, Lang T, Südhof TC (2003) Membrane fusion. Cell 112: 519-533

Jahn R, Scheller RH (2006) SNAREs-engines for membrane fusion. Nat Rev Mol Cell Biol 7: 631-643 
Lang T, Margittai M, Hölzler H, Jahn R (2002) SNAREs in native plasma membranes are active and readily form core complexes with endogenous and exogenous SNAREs. J Cell Biol 158: $751-760$

Low SH, Vasanji A, Nanduri J, He M, Sharma N, Koo M, Drazba J, Weimbs $T$ (2006) Syntaxins 3 and 4 are concentrated in separate clusters on the plasma membrane before establishment of cell polarity. Mol Biol Cell 17: 977-989

Maxfield FR, McGraw TE (2004) Endocytic recycling. Nat Rev Mol Cell Biol 5: 121-132

Peng R, Gallwitz D (2002) Sly1 protein bound to Golgi syntaxin Sed5p allows assembly and contributes to specificity of SNARE fusion complexes. J Cell Biol 157: 645-655

Pobbati AV, Stein A, Fasshauer D (2006) N- to C-terminal SNARE complex assembly promotes rapid membrane fusion. Science $\mathbf{3 1 3}$ : 673-676

Prekeris R, Klumperman J, Chen YA, Scheller RH (1998) Syntaxin 13 mediates cycling of plasma membrane proteins via tubulovesicular recycling endosomes. J Cell Biol 143: 957-971

Rizzoli SO, Bethani I, Zwilling D, Wenzel D, Siddiqui TJ, Brandhorst D, Jahn R (2006) Evidence for early endosome-like fusion of recently endocytosed synaptic vesicles. Traffic 7: $1163-1176$

Shen J, Tareste DC, Paumet F, Rothman JE, Melia TJ (2007) Selective activation of cognate SNAREpins by Sec1/Munc18 proteins. Cell 128: $183-195$
Sieber JJ, Willig KI, Heintzmann R, Hell SW, Lang T (2006) The SNARE motif is essential for the formation of syntaxin clusters in the plasma membrane. Biophys $J$ 90: 2843-2851

Stenmark H, Parton RG, Steele-Mortimer O, Lutcke A, Gruenberg J, Zerial M (1994) Inhibition of rab5 GTPase activity stimulates membrane fusion in endocytosis. EMBO J 13: 1287-1296

Sun W, Yan Q, Vida TA, Bean AJ (2003) Hrs regulates early endosome fusion by inhibiting formation of an endosomal SNARE complex. J Cell Biol 162: 125-137

Takamori S, Holt M, Stenius K, Lemke EA, Grønborg M, Riedel D, Urlaub H, Schenck S, Brugger B, Ringler P, Muller SA, Rammner B, Grater F, Hub JS, De Groot BL, Mieskes G, Moriyama Y, Klingauf J, Grubmüller $\mathrm{H}$, Heuser $\mathrm{J}$ et al (2006) Molecular anatomy of a trafficking organelle. Cell 127: 831-846

Varlamov O, Volchuk A, Rahimian V, Doege CA, Paumet F, Eng WS, Arango N, Parlati F, Ravazzola M, Orci L, Söllner TH, Rothman JE (2004) i-SNAREs: inhibitory SNAREs that fine-tune the specificity of membrane fusion. J Cell Biol 164: 79-88

Wang L, Merz AJ, Collins KM, Wickner W (2003) Hierarchy of protein assembly at the vertex ring domain for yeast vacuole docking and fusion. J Cell Biol 160: 365-374

Zhang T, Hong W (2001) Ykt6 forms a SNARE complex with syntaxin 5, GS28, and Bet1 and participates in a late stage in endoplasmic reticulum-Golgi transport. J Biol Chem 276: 27480-27487

Zilly FE, Sørensen JB, Jahn R, Lang T (2006) Munc18-bound syntaxin readily forms SNARE complexes with synaptobrevin in native plasma membranes. PLoS Biol 4: e330 